\title{
Knowledge, Attitudes and Perceptions Regarding Endemic Vivax Malaria in Inhabitants and Patients in Two Cities of Northern Gyeonggi-do, Korea, 2020
}

\author{
Young Yil Bahk ${ }^{10}$, Shin-Hyeong Cho ${ }^{2}$, Sookkyung Park ${ }^{3}$, Jeongran Kwon ${ }^{3}$, Hyesu Kan ${ }^{3}$, Miyoung Kim³, \\ Byoung-Kuk Na ${ }^{4}$, Sung Jong Hong ${ }^{5}$, Hyung Wook Kwon ${ }^{5,6, *}$, Tong-Soo Kim ${ }^{5, *}$ (]) \\ 'Department of Biotechnology, College of Biomedical and Health Science, Konkuk University, Chungju 27478, Korea; ' 2 Noul Co. LTD., Yongin 16942, \\ Korea; ${ }^{3 B}$ Bureau of Infectious Disease Policy, Korea Disease Control and Prevention Agency, Osong 28159, Korea; ${ }^{4}$ Department of Parasitology and \\ Tropical Medicine, Institute of Health Sciences, Gyeongsang National University College of Medicine, and Department of Convergence Medical \\ Science, Gyeongsang National University, Jinju 52727, Korea; ${ }^{5}$ Convergence Research Center for Insect Vectors, Incheon National University, \\ Incheon 22012, Korea; ' $D$ Department of Biological Science and Center for Insect Vectors, Incheon National University, Incheon 22012, Korea
}

\begin{abstract}
An understanding of the knowledges, attitudes and perceptions of different populations is key for public health policy makers. Here, a survey was performed on knowledge, attitudes, and perceptions about malaria diagnosis, prevention, control, and treatment. The 407 survey participants included both uninfected inhabitants and patients from 2 cities (Gimpo- and Paju-si) of Northern Gyeonggi-do, known as high-risk areas for vivax malaria. We used community-based study design and non-probability sampling method using the primary data. Association between variables were tested using $\chi^{2}$-tests. In general, the information on malaria reported by the participants in this study was unsystematic and included inaccurate details. The knowledge of malaria symptoms, identified as headache, chills and fever, was high, but the surveyed community lacks knowledge of the specific medications used for malaria treatment, with a large number of respondents having no knowledge of any form of medication. Survey questions with high correct answer rates included questions about easy treatment of malaria in Korea, the high daytime activity of malaria-borne mosquitoes, and the infection risk posed by outdoor activities. However, a large portion of the respondents was unable to provide simple medical and biological information about the disease. This study aimed to comprehensively evaluate the knowledge, attitude, and practical behavior of the surveyed community with respect to malaria and the implications reported here could be applicable to other malaria endemic areas in Korea.
\end{abstract}

Key words: Vivax malaria, malaria knowledge, attitude, perception, questionnaire

\section{INTRODUCTION}

Malaria is an infectious mosquito-borne disease caused by protozoan parasites of genus Plasmodium. In the Republic of Korea (Korea), malaria has now been critically reduced to a plateau level (an incidence rate of approximately 1 per 100,000) [1]. Currently, it is expected that Korea will be able to successfully eliminate vivax malaria through the use of anti-malaria drugs, control of the vector and mass chemoprophylaxis. However, achieving elimination still remains a significant public health objective. Vivax malaria in Korea peaked at 4,142

- Received 20 August 2021, revised 22 November 2021, accepted 22 November 2021.

*Corresponding authors (hwkwon@inu.ac.kr; tongsookim@gmail.com) (c) 2021. Korean Society for Parasitology and Tropical Medicine This is an Open Access article distributed under the terms of the Creative Commons Attribution Non-Commercial License (https://creativecommons.org/licenses/by-nc/4.0) which permits unrestricted non-commercial use, distribution, and reproduction in any medium, provided the original work is properly cited. cases in 2000 and the current rate is approximately 500 cases per year $[1,2]$. Despite being preventable and treatable, vivax malaria continues to have a pivotal impact on individual's health near the regions adjacent to the demilitarized zone (DMZ) between South and North Korea [3].

Public awareness about the diagnosis, treatment, and prevention of vivax malaria is essential in preventing malaria infections and ultimately eliminating malaria [4]. In fact, the success of malaria elimination is highly dependent on the level of understanding in the local community, along with its attitudes and social characteristics [5]. Vivax malaria is a treatable and preventable disease requiring well-organized public and social programs. The World Health Organization (WHO) has recommended that Korea eliminate vivax malaria by 2023 [6], and the Korean Disease and Control and Prevention Agency (KDCA) has launched a 'Five-Year Action Plan for Malaria Elimination (2019-2023)'. However, the elimination of 
vivax malaria from Korea has proceeded slowly and has only been partially successful. In order to achieve this goal, in addition to diagnosing infections using appropriate tools and treating diagnosed patients with appropriate drugs, it is necessary to educate the community about malaria diagnosis, treatment, and prevention. Addressing community knowledge, attitudes, and perceptions is essential to designing and establishing sustainable and effective community-based malaria elimination strategies that will lead to behavior change and acclimation to new technology and ideas $[7,8]$.

Our study aimed to evaluate knowledge, attitudes and practices related to vivax malaria among uninfected inhabitants and symptomatic patients in high-risk areas (Gimpo- and Paju-si) of Northern Gyeonggi-do. There is a scarcity of research on this important issue for vivax malaria elimination in Korea. In fact, some previous studies have revealed that a population's knowledge of malaria is influenced by socio-demographic characteristics such as education status, residence, age, occupation, gender, and family monthly income $[9,10]$. Knowing that malaria diagnosis, treatment, prevention, and knowledge about mosquito vectors protect inhabitants' health and improve the public health situation, there is a need to encourage inhabitants and patients in high-risk regions to participate in and improve education on malaria prevention and treatment in order to accelerate progress towards the elimination of vivax malaria in Korea.

\section{MATERIALS AND METHODS}

\section{Ethical statement}

The study procedures concerning human sample collection, laboratory investigation, interviews, and questionnaire use were reviewed and approved by the Institutional Review Board (IRB) of Inha University (Approval No. 2020-04-004), and the study was conducted according to the principles expressed in the 1964 Helsinki Declaration. Before enrolment into the study, the objectives of the study and study procedures to be followed were explained to the participants, including both the uninfected inhabitants and the vivax malaria patients. Written informed consent was sought from all study participants, who were informed of their right to refuse to participate and/or to withdraw from the study at any time.

\section{Study area and period}

This study was conducted in 2 cities (Gimpo- and Paju-si) in Northern Gyeonggi-do, Korea between May and October 2020 (Fig. 1). These 2 cities adjacent to DMZ are known as the highrisk areas for malaria. According to the Statistics Korea, the total population size of these 2 cities is approximately one million. The 2 cities share the Han River estuary. The high-risk ar-

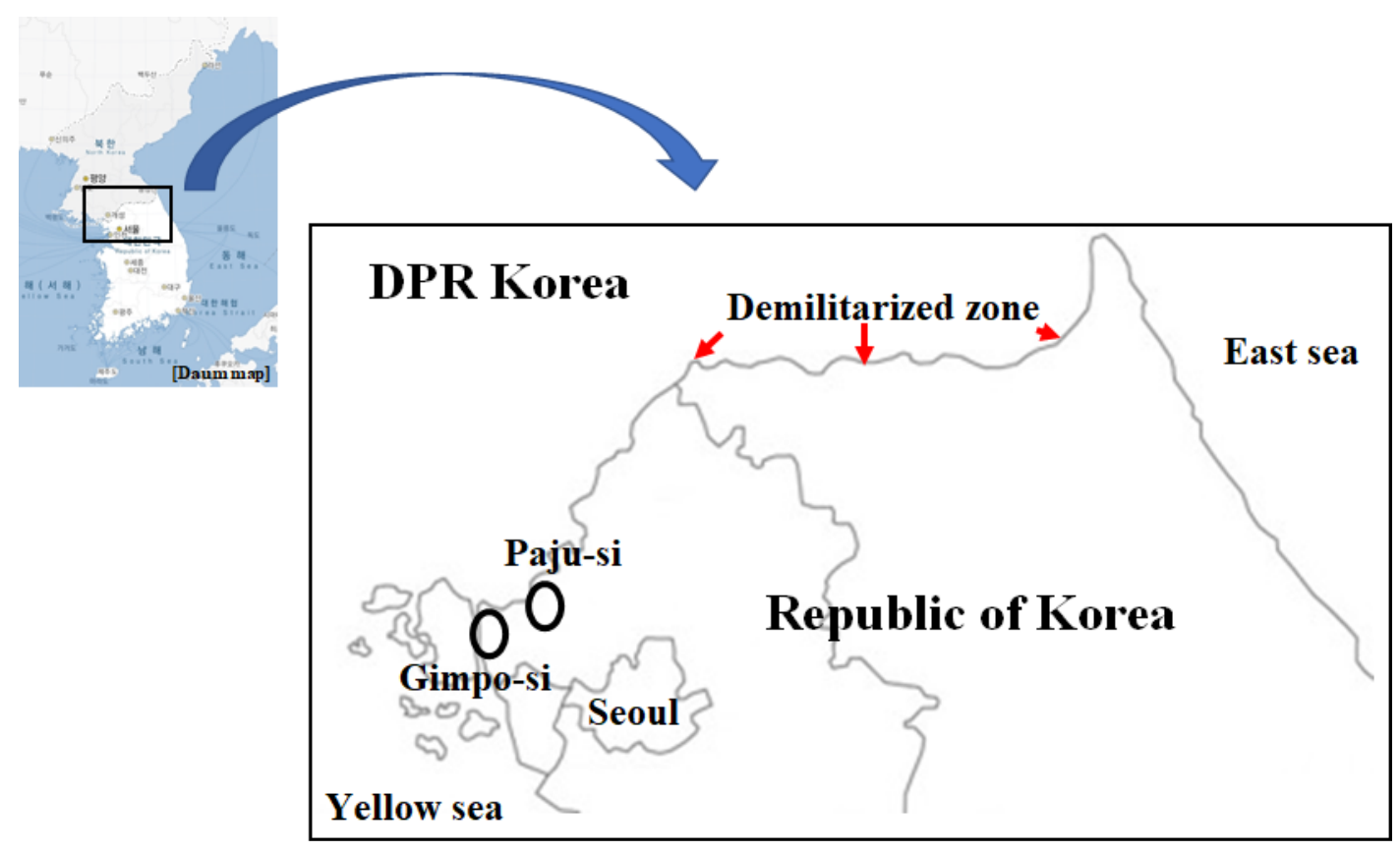

Fig. 1. Map of Gimpo-si and Paju-si, Gyeonggi-do: endemic regions of vivax malaria in Korea. 
eas in Korea that are adjacent to malaria-risk areas in North Korea, including Incheon-si, Gyeonggi-do, and Gangwon-do, experience over $70 \%$ of all vivax malaria cases in Korea [1]. Specifically, Gyeonggi-do experienced $46.0 \%$ of all vivax malaria cases in Korea from 2001 to 2020 (Fig. 2) [2].

\section{Study setting}

To collect relevant data, an interviewer-administered questionnaire was adapted from multiple previous studies. In this study, a total of 406 participants, including 380 uninfected inhabitants and 26 vivax malaria patients who were diagnosed and confirmed by the gold-standard microscopic techniques and PCR method, were interviewed, the collected data were analyzed. The questionnaire was originally prepared in Korean and collected 6 socio-demographic characteristics: gender, age, location of residence, occupation, residence type, and residence period (Table 1). The data were collected via face-to-face interviews by a diploma holder under the guidance of a supervisor. Training on data collection procedures and research ethics was given to data collectors and supervisors. The data collection process was closely supervised on a daily basis and feedback was given promptly.

\section{Data collection}

The dependent variables of this study were as follows:

The cognition level for vivax malaria was assessed using 12 malaria-cognition-related questions. The questions used to assess this knowledge addressed 1) knowing about vivax malaria, 2) the source of that information, 3) cognition status regarding malaria outbreaks in their residential area, 4) malaria infection status or history of oneself or acquaintance, 5) the malaria-causing agent, 6) the vector for transmission, 7) signs/ symptoms of malaria, 8) where malaria can be diagnosed 9) malaria prevention and treatment, 10) what can be used to prevent malaria, 11) knowledge about malaria therapy and 12) timing of malaria outbreaks. Multiple choices could be selected for some questions in the questionnaire.

Questions about the features of vivax malaria in Korea consisted of 13 questions about the status of vivax malaria in Korea, the therapeutic situation, the pattern of vivax malaria outbreaks in Korea, the transmission vector, the life cycle of the vector mosquitoes, the incubation period, and immunity. The 13 questions about malaria were scored at 7.6923 each, giving a total score of 100 and were calculated for each respondent.

Risk awareness of vivax malaria infection was assessed using 5 questions related to the risk level of vivax malaria and the effectiveness of public awareness efforts. The questions used to assess the risk awareness for vivax malaria regarded 1) the recognition of malaria risk in local residential areas, 2) whether the damage caused by the risk of malaria can be controlled through personal efforts, 3 ) the possible effects of malaria risk factors on family members and children, 4) fear of malaria infection risk, and 5) the seriousness of the consequences of malaria infection.

\section{Data analysis}

After the surveys were completed unanswered and dependent data were excluded from the analysis and the statistical package, SPSS version 21.0 (Statistical Package for Social Science, IBM, Armonk, New York, USA) and RStudio, were used

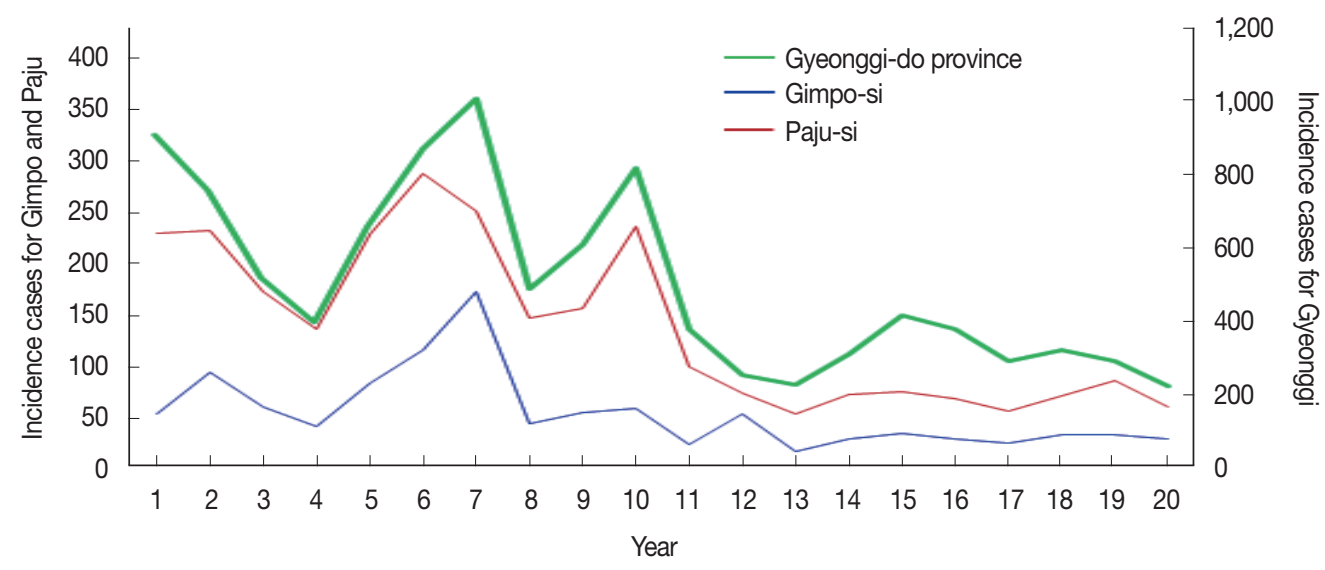

Fig. 2. Overall distribution of the incidence of vivax malaria in Gyeonggi-do, Gimpo-si, and Paju-si from 2001 to 2020 as reported by the KDCA in Korea. 
Table 1. Socio-demographic characteristics of respondents (uninfected inhabitants and patients) in the cities of Gimpo and Paju, Northern Gyeonggi-do, Korea, $2020(n=407)$

\begin{tabular}{|c|c|c|c|c|}
\hline \multirow[b]{2}{*}{ Variable } & \multirow[b]{2}{*}{ Category } & \multicolumn{2}{|c|}{ Frequency (\%) } & \multirow[b]{2}{*}{ Remarks } \\
\hline & & $\begin{array}{c}\text { Uninfected } \\
\text { inhabitant group }\end{array}$ & Patient group & \\
\hline \multirow[t]{2}{*}{ Gender } & Male & $141(37.1)$ & $19(73.1)$ & 1 response missing in uninfected inhabitant group \\
\hline & Female & $239(62.9)$ & 7 (26.9) & \\
\hline \multirow[t]{4}{*}{ Age } & $10 s-20 s$ & $26(7.6)$ & $11(42.3)$ & 38 responses missing in uninfected inhabitant group \\
\hline & $30 s-40 s$ & $80(23.3)$ & $9(34.6)$ & \\
\hline & $50 s-60 s$ & $141(41.1)$ & $4(15.4)$ & \\
\hline & $>70$ & $96(28.0)$ & $2(7.7)$ & \\
\hline \multirow[t]{2}{*}{ Residence } & Uninfected inhabitants* & $380(100)$ & 0 & \\
\hline & Patients & $0(0)$ & $26(100)$ & \\
\hline \multirow[t]{11}{*}{ Occupation } & Student & $12(4.4)$ & 8 (30.8) & 136 responses missing in uninfected inhabitant group \\
\hline & Housewife & $88(32.5)$ & $4(15.4)$ & \\
\hline & Primary industry worker & $56(20.7)$ & $1(3.8)$ & \\
\hline & Office worker & $22(8.1)$ & $2(7.7)$ & \\
\hline & Self-employed & $30(11.1)$ & $0(0)$ & \\
\hline & Labor position & $7(2.6)$ & $5(19.2)$ & \\
\hline & Service industry & $7(2.6)$ & $0(0)$ & \\
\hline & Academic profession & $12(4.4)$ & $1(3.8)$ & \\
\hline & Specialized job & $4(1.5)$ & $3(11.5)$ & \\
\hline & Annuitant & $11(4.1)$ & $0(0)$ & \\
\hline & The others & $22(8.1)$ & $2(7.7)$ & \\
\hline \multirow[t]{3}{*}{ Residence type } & Detached house & $191(50.8)$ & 5 (19.2) & 5 responses missing in uninfected inhabitant group \\
\hline & Apartment & $172(45.7)$ & $19(73.1)$ & \\
\hline & Multi-household house & $13(3.5)$ & $2(7.7)$ & \\
\hline \multirow[t]{5}{*}{ Residence period } & $<10$ years & $88(24.0)$ & $7(87.5)$ & 15 responses missing in uninfected inhabitants \\
\hline & $\geq 10-<30$ years & $128(35.0)$ & $1(12.5)$ & 18 responses missing in patient group \\
\hline & $\geq 30-<50$ years & $72(19.7)$ & 0 & \\
\hline & $\geq 50-<70$ years & $58(15.8)$ & 0 & \\
\hline & $\geq 70$ years & $20(5.5)$ & 0 & \\
\hline
\end{tabular}

*Malaria model areas: Siam-ri (Gimpo-si), Bongilcheon-ri, Jangpa-ri, and Unjeong district (Paju-si).

to perform the data coding and cleaning processes. The statistical procedures in this study were performed by ST-Research, Inc. (Busan, Korea). Results for categorical variables were reported using descriptive statistics such as frequencies and percentages. The variables analyzed included knowledge of malaria, sociodemographic factors, malaria related knowledge, attitudes, and practices. Frequencies were compared using the chi-squared $\left(\chi^{2}\right)$ test. Mean and standard deviation were computed for numerical variables. To assess the knowledge of respondents about malaria, answers to the questions concerning how malaria is transmitted and the ability to identify mosquitoes as a vector of malaria were considered. Participants providing correct answers to the questions were considered as having good knowledge of malaria transmission. In addition, those questions concerning good practices in regard to malaria prevention and treatment were assessed.

\section{RESULTS}

\section{Socio-demographic characteristics}

Table 1 presents the socio-demographic characteristics of the participants in this study. Among the 407 respondents, 381 uninfected inhabitants were from the Siam-ri (district) of Gimpo-si and the Bongilcheon-ri, Jangpa-ri, and Unjeong district of Paju-si, which have been designated as malaria model areas. The participants consisted of 141 males (37.1\%) and 239 females (62.9\%) and 141 participants (41.1\%) were in the age group of 50s-60s age group. The mean age of all participants was 57.8 years. The most common occupation of the participants was housewife $(32.5 \%)$, followed by primary industry worker (20.7\%) and self-employed (11.1\%). The housing types of the respondents were detached house (50.8\%), apartment (45.7\%), and multi-household house (3.5\%). 
Twenty-six study participants were vivax malaria patients, consisting of 19 males (73.1\%) and 7 females (26.9\%). The patients were from Gimpo-si, and their mean age was 38.0 years. The main age group was $10 \mathrm{~s}-20 \mathrm{~s}(42.3 \%)$, followed by the 30s-40s group (34.6\%).

\section{Study respondents' knowledge of malaria transmission and symptoms}

Study respondents were asked if they have ever heard of malaria to which 352 responded positively (uninfected inhabitant group; UI group): 332/375, patient group (P group): $76.9 \%$ ) (Table 2). The most common sources of malaria-related information were TV and radio (204/386, UI group: 194/360, P group: 10/26), family members or neighbors (83, UI group: 81, P group: 2), health facilities (hospitals or drug stores, 50, UI group: 43, P group: 7), and mobile and internet news (30, UI group: 27, P group: 3). Most study participants (334/398, UI group: 308/372, P group: 26/26) were aware that the vector for malaria transmission is mosquitoes. However, only 39 (UI group: 28/370, P group: 11/26) identified a reported parasite as the disease-causing agent, while 189 (UI group: 187, P group: 2) reported that it was an insect, and 70 (UI group: 64, P group: 6) did not know $\left(\chi^{2}=10.751, P<0.05\right)$. The 3 most commonly mentioned symptoms of malaria were fever or chills (323/401, UI group: 297/375, P group: 26/26), muscle pain (75, UI group: 59, P group: 16/26), and headache (47, UI group: 31, P group: 16). The study further assessed the knowledge of participants about malaria diagnosis, prevention, and treatment measures. Regarding where one can be diagnosed, most of the participants responded with hospitals (188/401, UI group: 163/375, P group: 25/26) and public facilities (290, UI group: 276, P group: 14). The majority of respondents thought that they should go to a health center/clinic to confirm malaria. However, knowledge on the preventive and therapeutic measures was relatively limited; $49.3 \%$ of the respondents (198/402, UI group: 188/376, P group: 10/26) reported that they did not know about methods for the prevention and treatment of malaria. Among the remaining respondents, the 3 commonly reported preventive measures were not getting bitten by mosquitoes (220/401, UI group: 199/375, P group: 21/26), wearing long sleeved and bright clothing (151, UI group: 138, P group: 13), and being cautious when visiting the malaria risk areas (155, UI group: 135, P group: 10). A comparison of the responses about malaria prevention measures showed that the difference in responses be- tween the UI and P groups was statistically significant $\left(\chi^{2}=\right.$ $17.649, P<0.05)$. Of the respondents, $47.8 \%(192 / 402$, UI group: $45.5 \%$ ), P group: $80.8 \%$ ) reported that for the treatment of malaria, one should take the whole dosage of hospital-prescribed medication, while $35.8 \%$ (UI group: $37.0 \%$, P group: $19.2 \%$ ) had no knowledge to report about prescription medication. Regarding the timing of malaria outbreaks, approximately 3 quarters of study respondents (75.1\%, UI group: $74.1 \%$, P group: $23 / 26$ ) said that the summer season is the major malaria outbreak time.

\section{Respondents' attitudes and knowledge about vivax malaria}

In this study we assessed study participants' attitudes towards malaria (Table 3). An overall knowledge score was calculated for each participant. The 13 questions about the features of vivax malaria were scored at 7.6923 each, giving a total score out of 100 that was calculated for each respondent. Most study respondents (83.5 points; UI group: 83.1, P group: 88.5) agreed that participating in many outdoor activities has a strong effect on malaria risk. Most study respondents also gave the correct answers that malaria that Korea has high-quality treatment for malaria (86.6 points; UI group: 80.4, P group: 92.3 ) and that malaria-bearing mosquitoes are mostly active during the daytime (86.6 points; UI group: 80.1, P group: 96.2). Some misconceptions regarding malaria were also reflected in negative responses to questions about attitude. Only a small portion of the study respondents (22.9 points; UI group: 21.6, P group: 42.3) gave the correct answer that Korea has the highest malaria infection rate among the Organization for Economic Cooperation and Development (OECD) countries. In terms of malaria treatment, approximately half of the respondents (55.5 points; UI group: 54.7, P group: 69.2) correctly stated that vivax malaria, which is prevalent in Korea, can be fatal if untreated. Similarly, approximately half of the study respondents (55.5 points; UI group: 50.1, P group: 80.8) reported that the malaria that occurs in Korea is transmitted by female mosquitoes. Fewer of the uninfected inhabitants were familiar with the long incubation period of vivax malaria relative to the patient group (58.2 vs. 80.8 points, respectively). Around one quarter of the study respondents (27.8 points) said that malaria infections can be prevented through malaria vaccines. Among the uninfected inhabitants' scores for the question about malaria characteristics ranged from 0 to 100 with an average of $59.3(\mathrm{SD} \pm 17.9)$. In contrast, in the patient 
Table 2. Knowledge and recognition of Rrespondent's cognition towards vivax malaria

\begin{tabular}{|c|c|c|}
\hline \multirow{2}{*}{ Question } & \multicolumn{2}{|c|}{ Frequency (\%) } \\
\hline & Uninfected inhabitant group & Patient group \\
\hline Knowing vivax malaria & $n=375$ & $\mathrm{n}=26$ \\
\hline Never known & $7(1.9)$ & $3(1.5)$ \\
\hline Unknown & $36(9.6)$ & $3(1.5)$ \\
\hline Heard & $150(40.0)$ & $13(50.0)$ \\
\hline Known & $139(37.1)$ & $3(11.5)$ \\
\hline Well known & $43(11.5)$ & $4(15.5)$ \\
\hline Source of information ${ }^{\star}$ & $n=360$ & $n=26$ \\
\hline TV and Radio & $194(53.9)$ & $10(38.5)$ \\
\hline Mobile and internet news & $27(7.5)$ & $3(11.5)$ \\
\hline Internet (Café or Blog etc.) & $16(4.4)$ & $4(15.4)$ \\
\hline SNS (Facebook, Kakaostory, etc.) & $6(1.7)$ & $1(3.8)$ \\
\hline Newspaper or magazine & $15(4.2)$ & $1(3.8)$ \\
\hline Publicity materials (Poster etc.) & $16(4.4)$ & $0(0)$ \\
\hline Health facility (Hospital or drug store) & $43(11.9)$ & $7(26.9)$ \\
\hline Family member or neighbors & $81(22.5)$ & $2(7.7)$ \\
\hline The others & $30(8.3)$ & $2(7.7)$ \\
\hline Malaria outbreak in their residential areas & $n=376$ & $n=26$ \\
\hline Unknowing & $111(29.5)$ & $12(46.2)$ \\
\hline Knowing & $265(70.5)$ & $14(953.8)$ \\
\hline Malaria infection status or history of oneself or acquaintance & $n=373$ & $n=26$ \\
\hline No & $249(66.8)$ & $0(0)$ \\
\hline Yes & $124(33.2)$ & $26(100.0)$ \\
\hline Disease-causing agent & $n=370$ & $n=26$ \\
\hline Bacteria & $27(7.3)$ & $2(7.7)$ \\
\hline Virus & $64(17.3)$ & $5(19.2)$ \\
\hline Parasite & $28(7.6)$ & $11(42.3)$ \\
\hline Insect & $187(50.5)$ & $2(7.7)$ \\
\hline I don't know & $64(17.3)$ & $6(23.1)$ \\
\hline Vector for transmission & $n=372$ & $n=26$ \\
\hline Mite & $17(4.6)$ & 0 \\
\hline Fly & $4(1.1)$ & 0 \\
\hline Mosquito & $308(82.8)$ & $26(100.0)$ \\
\hline Mayfly & $0(0)$ & 0 \\
\hline Mouse or rate & $5(1.3)$ & 0 \\
\hline I don't know & $38(10.2)$ & 0 \\
\hline Signs/symptoms of malaria* & $n=375$ & $n=26$ \\
\hline Fever or chills & $297(79.2)$ & $26(100)$ \\
\hline Muscle pain & $59(15.7)$ & $16(61.5)$ \\
\hline Cough and throat ache & $16(4.3)$ & $0(0)$ \\
\hline Headache & $31(8.3)$ & $16(61.5)$ \\
\hline Diarrhea & $33(8.8)$ & $2(7.7)$ \\
\hline I don’t know & $54(14.4)$ & $0(0)$ \\
\hline Where malaria can be diagnosed* & $n=375$ & $n=26$ \\
\hline Hospital & $163(43.5)$ & $25(96.2)$ \\
\hline Public health facilities & $276(73.6)$ & $14(53.8)$ \\
\hline Oriental medical clinic & $0(0)$ & $0(0)$ \\
\hline Drug store & $3(0.8)$ & $0(0)$ \\
\hline I don't know & $16(4.3)$ & $1(3.8)$ \\
\hline
\end{tabular}


Table 2. Continued

\begin{tabular}{|c|c|c|}
\hline \multirow{2}{*}{ Question } & \multicolumn{2}{|c|}{ Frequency (\%) } \\
\hline & Uninfected inhabitant group & Patient group \\
\hline Recognition for malaria prevention and treatment & $n=376$ & $n=26$ \\
\hline Only malaria prevention & $105(27.9)$ & $4(15.4)$ \\
\hline Only malaria treatment & $10(2.7)$ & $3(11.5)$ \\
\hline Both prevention and treatment & $73(19.4)$ & $9(34.6)$ \\
\hline I don’t know & $188(50.0)$ & $10(38.5)$ \\
\hline The following can be used to prevent malaria* & $\mathrm{n}=375$ & $n=26$ \\
\hline Not getting bitten by mosquitoes & $199(53.1)$ & $21(80.8)$ \\
\hline Spraying insecticide or repellent agent & $110(29.3)$ & 9 (34.6) \\
\hline Wearing long sleeved and bright clothing & $138(36.8)$ & $13(50.0)$ \\
\hline Refraining from going out during mosquito activity & $113(30.1)$ & 7 (26.9) \\
\hline Vaccination every year & $67(17.9)$ & $1(3.8)$ \\
\hline Use of mosquito net & $114(30.4)$ & $4(15.4)$ \\
\hline Being cautious when visiting malaria risk area & $135(36.1)$ & $10(38.5)$ \\
\hline I don’t know & $67(17.9)$ & $2(7.7)$ \\
\hline Knowledge about malaria therapy & $n=376$ & $n=26$ \\
\hline One dosage of hospital prescription medication & $15(4.0)$ & $0(0)$ \\
\hline Whole dosage of hospital prescription medication & $171(45.5)$ & $21(80.8)$ \\
\hline One injection at the hospital & $65(17.3)$ & $0(0)$ \\
\hline Can't cure malaria & $7(1.9)$ & $0(0)$ \\
\hline I don’t know & $139(37.0)$ & $5(19.2)$ \\
\hline Time of malaria outbreaks & $n=344$ & $n=26$ \\
\hline Winter (Dec.-Mar.) & $0(0)$ & $0(0)$ \\
\hline Autumn (Sep.-Nov.) & $23(6.7)$ & $1(3.8)$ \\
\hline Summer (Jun.-Aug.) & $255(74.1)$ & $23(88.5)$ \\
\hline Spring (Apr.-May) & $12(3.5)$ & $0(0)$ \\
\hline Year-round & $30(8.7)$ & $2(7.7)$ \\
\hline I don't know & $24(7.0)$ & $0(0)$ \\
\hline
\end{tabular}

${ }^{*}$ Respondents could choose multiple answers.

Table 3. Respondent's answers to the questions about vivax malaria characteristics in Korea

\begin{tabular}{|c|c|c|}
\hline \multirow[b]{2}{*}{ Question } & \multicolumn{2}{|c|}{ Correct answer rate } \\
\hline & $\begin{array}{l}\text { Uninfected inhabitant } \\
\text { group }(\mathrm{n}=381)\end{array}$ & $\begin{array}{l}\text { Patient group } \\
\quad(n=26)\end{array}$ \\
\hline Korea has the highest malaria infection rate among OECD countries. & 21.6 & 42.3 \\
\hline Malaria that occurs in Korea is well treated. & 80.4 & 92.3 \\
\hline Malaria-bearing mosquitoes are mostly active during the daytime. & 80.1 & 96.2 \\
\hline Malaria, which is prevalent in Korea, can be fatal if untreated. & 54.7 & 69.2 \\
\hline Malaria can also occur in early spring or late autumn when vector mosquitoes are not active. & 75.0 & 76.9 \\
\hline $\begin{array}{l}\text { Individuals who take part in many outdoor activities such as fishing and outdoor sports can easily } \\
\text { become infected with malaria }\end{array}$ & 83.1 & 88.5 \\
\hline If infected with malaria, it can stay hidden in the liver for at least two weeks and up to 24 months. & 58.2 & 80.8 \\
\hline $\begin{array}{l}\text { Recovered patients can be reinfected with malaria if bitten by mosquitoes even after treatment is } \\
\text { completed. }\end{array}$ & 71.0 & 92.3 \\
\hline Malaria infections can be prevented by malaria vaccines. & 24.9 & 73.1 \\
\hline All species of mosquitoes living in Korea can transmit malaria. & 68.0 & 92.3 \\
\hline Malaria can be transmitted through blood donation. & 67.9 & 84.6 \\
\hline Malaria occurring in Korea is transmitted by female mosquitoes. & 50.1 & 80.8 \\
\hline After getting malaria and recovering completely, immunity against malaria is sustained for life. & 77.5 & 96.2 \\
\hline
\end{tabular}


group, the minimum score was 53.8 and the maximum score was 100 with an average of 81.9 ( $S D \pm 18.7$ ). The difference in scores between the UI and P groups is statistically significant $(P<0.05)$ (Fig. 3).

\section{Respondents' risk awareness of malaria infection}

We assessed the study respondents' awareness of the risks associated with malaria infection (Table 4). Respondents were asked about the malaria risk in their local residential areas. Approximately $22 \%$ of the respondents, either agreed (14.5\%) or strongly agreed (7.8\%) that their local residential areas are secure against malaria infection, whereas $33.8 \%$ of the respondents did not agree that their area was secure against malaria. Half of the respondents said they were convinced that the health risk posed by malaria can be controlled through personal efforts. However, only $23.1 \%$ in the P group felt that way. Also, $59.1 \%$ of the study respondents either agreed (48.1\%) or

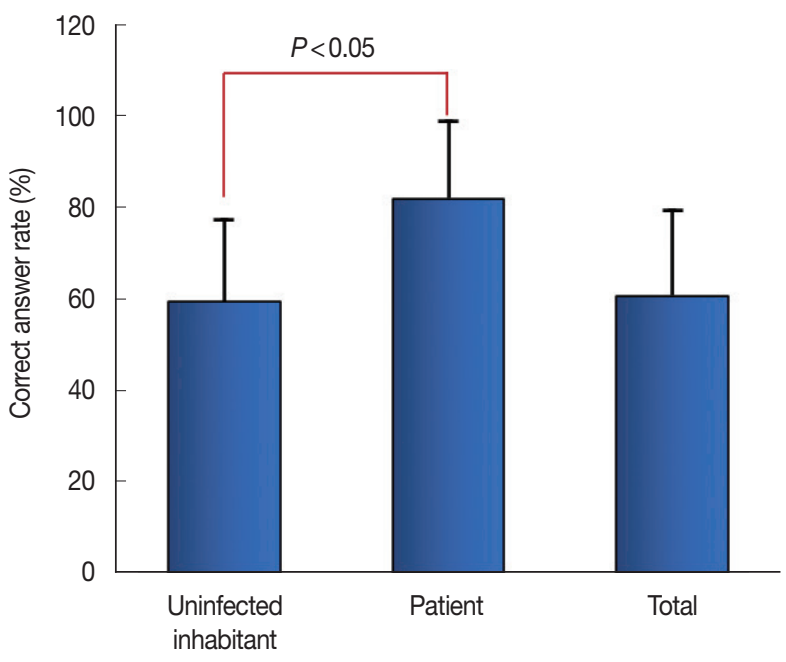

Fig. 3. Respondents' correct answer rates to the questions about malaria characteristics in Korea. The 13 questions about malaria were scored at 7.6923 points each, giving a total possible score of 100 that was calculated for each respondent. The difference in positive scores between the $\mathrm{UI}$ group and the $\mathrm{P}$ group is statistically significant $(P<0.05)$.

Table 4. Resident awareness of malaria infection risks

\begin{tabular}{|c|c|c|}
\hline \multirow{2}{*}{ Question } & \multicolumn{2}{|c|}{ Frequency (\%) } \\
\hline & Uninfected inhabitant group & Patient group \\
\hline Recognition of malaria risk in local residential areas & $n=373$ & $n=26$ \\
\hline Completely not secure & $46(12.3)$ & $1(3.8)$ \\
\hline Not secure & $73(19.6)$ & $15(57.7)$ \\
\hline Being average in security & $169(45.3)$ & $6(23.1)$ \\
\hline Secure & $54(14.5)$ & $4(15.4)$ \\
\hline Absolutely secure & $31(8.3)$ & $0(0)$ \\
\hline Can the risk of damage caused by malaria be controlled through personal efforts? & $n=373$ & $n=26$ \\
\hline Completely impossible & $35(9.4)$ & $1(3.8)$ \\
\hline Impossible & $70(18.8)$ & $14(53.8)$ \\
\hline Being average & $75(20.1)$ & $5(19.2)$ \\
\hline Possible & $169(45.3)$ & $4(15.4)$ \\
\hline Absolutely possible & $24(6.4)$ & $2(7.7)$ \\
\hline Possible effects of malaria risk factors on family members and children & $\mathrm{n}=371$ & $n=26$ \\
\hline Completely not influential & $25(6.7)$ & $2(7.7)$ \\
\hline Not influential & $61(16.4)$ & $0(0)$ \\
\hline Being average & $70(18.9)$ & $4(15.4)$ \\
\hline Influential & $173(46.6)$ & $18(69.2)$ \\
\hline Absolutely influential & $42(11.3)$ & $2(7.7)$ \\
\hline Fear of malaria infection risk & $n=371$ & $n=26$ \\
\hline Completely not scared & $33(8.9)$ & $0(0)$ \\
\hline Not scared & $74(19.9)$ & $4(15.4)$ \\
\hline Being average & $83(22.4)$ & $5(19.2)$ \\
\hline Scared & $136(36.7)$ & $14(53.8)$ \\
\hline Absolutely scared & $45(12.1)$ & $3(11.5)$ \\
\hline Seriousness of the consequences of malaria infection & $n=371$ & $\mathrm{n}=26$ \\
\hline Completely not serious & $25(6.7)$ & $0(0)$ \\
\hline Not serious & $54(14.6)$ & $0(0)$ \\
\hline Being average & $77(20.8)$ & $2(7.7)$ \\
\hline Serious & $167(45.0)$ & $21(80.8)$ \\
\hline Absolutely serious & $48(12.9)$ & $3(11.5)$ \\
\hline
\end{tabular}


strongly agreed (11.1\%) that malaria itself and the risks related to malaria infection will affect their family members and children. The respondents in the patient group were especially likely to agree with the statement $(76.9 \%)$. When the respondents were asked whether they are usually afraid of malaria and the risks that it poses, around half of the respondents (49.9\%) confirmed that they are either scared (37.8\%) or absolutely scared (12.1\%). In this measure, the respondents in the P group showed a greater tendency to be scared $(65.4 \%$ vs. $48.8 \%$ of the UI group). When looking at the fear of malaria infection, the averages in the UI group and the P groups were 3.23 and 3.62 out of 5, respectively. The difference between the averages for the 2 groups was statistically significant $(P<0.05)$. Lastly, $60.2 \%$ of the respondents, either agreed $(47.4 \%)$ or strongly agreed $(12.8 \%)$ that malaria is a serious infectious disease. When asked about how serious a health risk they considered malaria infection to be on a scale from 1 to 5 , the averages for the UI and P groups were 3.43 and 4.04 respectively. The average difference between the 2 groups was statistically significant $(P<0.05)$.

\section{DISCUSSION}

This study mainly aimed to confirm the level of knowledge, attitudes, and practices regarding malaria of communities living in high-risk areas. The respondents including both uninfected inhabitants and symptomatic patients, who were diagnosed with the gold standard method, in the cities of Gimpo and Paju, which are adjacent to the DMZ, Northern Gyeonggido. We have used a community-based study design and nonprobability sampling using the primary data, rather than a hospital- and health facility-based study design and probability sampling. A good fundamental knowledge of malaria among the population could make a significant contribution toward malaria elimination. Overall, we found needs for improvement in the residents' knowledge, attitudes and practices related to malaria prevention and control.

Study respondents had generally received broad information about malaria through TV/radio, family members and acquaintances, health facilities including hospitals or drug stores, and mobile/internet news. The source of information on malaria identified most often by the participants was TV and radio and the relatively least-mentioned source $(12.3 \%$ of the respondents) was health facilities including public health agencies, hospitals and drug stores. However, the information recalled by the respondents was unsystematic and included inaccurate details. In particular, it is noteworthy that the proportion of respondents in the patient group who had no knowledge of malaria was high. Therefore, it is deemed necessary to investigate focusing on this aspect in the near future. In other words, the results suggest that to improve the prevention and treatment of malaria in Korea, where the disease is endemic, policy makers should consider methods and tools to effectively promote accurate and practical knowledge about malaria, such as information about public healthcare facilities and prevention and treatment methods. The knowledge of malaria symptoms was high in this study. Most of the respondents correctly identified the 3 most common symptoms of malaria (headache, chills and fever). This coincides with the results of other studies indicating that people in malaria endemic areas are aware of the clinical manifestations of the disease [11,12]. However, incorporating local terminology and knowledge into malaria education and public health promotion may not be effective at changing community knowledge in the shortest possible time. The most common occupation among the study participants was housewife. In general, it is well established that educational status is a significant factor affecting the timing of seeking treatment for malaria $[4,13,14]$. Although, similar to other studies in malaria endemic settings [15-17], most study respondents were aware that the vector for malaria transmission is mosquitoes, a small portion $(9.8 \%)$ of study respondents identified the malaria disease-causing agent as a parasite, while $46.4 \%$ of the respondents believed that it is an insect. In addition, knowledge about preventive and therapeutic measures for malaria was relatively low. Approximately $2 / 3$ of the respondents in the UI group simply mentioned the ineffective medication for the treatment of malaria. This suggests that the studied community lacks knowledge of the specific details of the medications used for malaria treatment. Highlighting the finding, a large number of the respondents, even including participants in the P group, had no knowledge of any form of medication for malaria treatment (Table 2). Thus, it is proposed that education and propaganda that provides simple biological and medical information is less meaningful than other measures for improving malaria knowledge and that proactive, concise and in-depth discussions are necessary to convey this information to patients and the general population in the future.

In this study of respondents' attitudes towards the question of malaria characteristics in Korea, questions with high correct 
answer rates included those about the quality of malaria treatment in Korea, the high daytime activity level of malaria-bearing mosquitoes, and the infection risk involved in outdoor activities. This was evidence of the population's knowledge of preventive practices. Korea's malaria incidence rate is 1 in every 100,000 people, the highest rate among OECD countries, followed by Mexico with 0.6 and no endemic malaria in other OECD countries. In our study, awareness about the long-term incubation period of vivax malaria was relatively low (58.2 points for UI group and 80.8 for P group). Also, only approximately a quarter of respondents, especially in the UI group, were able to provide correct information about vaccines against vivax malaria. Unlike Plasmodium falciparum, the $P$. vivax life cycle includes a dominant liver stage, the hypnozoite. An effective vaccine against $P$. vivax blood stages would limit symptoms and pathology from such recurrent infections, but vaccine development for $P$. vivax lingers behind that for $P$. falciparum. Although a vaccine against vivax malaria could play a significant role in malaria control, there is currently no effective vaccine against the blood stage of any malaria parasite, and progress on $P$. vivax vaccine development has been particularly hampered [18]. The correct answer rate of the patient group for the question about vaccines was statistically significantly higher than that of the general population (Fig. 3).

Strikingly, study participants were less aware of the occurrence of malaria in their local area. Study participants were split about the impact that they believed malaria and its associated risks to have on their own home and their neighborhood. A little more than half of the responses (59.1\%) indicated that participants are aware that malaria infection poses risks to their health. Also, half of respondents (49.9\%) are afraid of malaria and its associated risks and $60.2 \%$ of the respondents consider malaria to have a very significant impact on their family members, including their children, and their neighborhood.

In general, this study has aimed to comprehensively access the knowledge, attitudes and practical behaviors of the community with regard to malaria prevention, diagnosis, treatment, and control. This study had notable impacts, including the collection of fundamental information on malaria knowledge, attitudes, perception, and practices in local communities as well as contribution to malaria awareness by providing participants with additional information on malaria. However, sustained and strong research is necessary in order to evaluate the effectiveness of public health campaigns against malaria as well as the motivation of community residents regarding awareness of malaria. The implications of this study could be extended to other malaria endemic areas in Korea, indicating a need to consider the aforementioned population characteristics in a national malaria elimination program.

\section{ACKNOWLEDGMENTS}

This study was supported by funding from the Korea Disease Control and Prevention Agency (KDCA), by funding from the National Research Fund (NRF-2020R1F1A1070882, 2018M3A9H5055614) in Korea and by the Priority Research Centers Program through the National Research Foundation of Korea (NRF) funded by the Ministry of Education (2020 R1A6A1A03041954).

\section{CONFLICT OF INTEREST}

The authors declare that they have no conflicts of interest regarding the publication of this article.

\section{REFERENCES}

1. Bahk YY, Lee HW, Na BK, Kim J, Jin K, Hong YS, Kim TS. Epidemiological characteristics of re-emerging vivax malaria in the Republic of Korea (1993-2017). Korean J Parasitol 2018; 56: 531543. http://doi.org/10.3347/kjp.2018.56.6.531

2. Korean Disease Control and Prevention Agency. Infectious Diseases Surveillance System [Internet]. Available from: https://is. kdca.go.kr/

3. Kim JH, Lim AY, Cheong HK. Malaria incidence of the regions adjacent to the demilitarized zone in the Democratic People's Republic of Korea, 2004-2016. J Korean Med Sci 2019; 34: e227. https://doi.org/10.3346/jkms.2019.34.e227

4. Kebede DL, Hibstu DT, Birhanu BE, Bekele FB. Knowledge, attitude and practice towards malaria and associated factors in Areka Town, Southern Ethiopia: commuUIty-based cross-sectional study. J Trop Dis 2017; 5: 240. https://doi.org/10.4172/2329891X. 1000240

5. Tynan A, Atkinson JA, Toaliu H, Taleo G, Fitzgerald L, Whittaker M, Riley I, Schubert M, Vallely A. CommuUIty participation for malaria elimination in Tafea Province, Vanuatu: part II. Social and cultural aspects of treatment-seeking behavior. Malar J 2011; 10: 204. https://doi.org/10.1186/1475-2875-10-204

6. World Health Organization. WHO Country Cooperation Strategy 2019-2023: Republic of Korea. World Health Organization Regional Office for the Western Pacific. 2019. https://apps.who. int/iris/handle/10665/279858.

7. Ruebush TK II, Zeissig R, Koplan JP, Klein RE, Godoy HA. Com- 
munity participation in malaria surveillance and treatment III. An evaluation of modifications in the volunteer collaborator network of Guatemala. Am J Trop Med Hyg 1994; 50: 85-98. https://doi. org/10.4269/ajtmh.1994.50.85

8. Adongo PB, Kirkwood B, Kendall C. How local commuUIty knowledge about malaria affects insecticide-treated net use in northern Ghana. Trop Med Int Health 2005; 10: 366-378. https:// doi.org/10.1111/j.1365-3156.2005.01361.x

9. Jimee H, Patricia MG, Jima D, Richard R, Kachu SP, The Ethiopia MIS Working Group. Knowledge of malaria and its association with malaria-related behaviors-Results from the malaria indicator survey, Ethiopia, 2007. PLoS One 2010; 5: e11692. https://doi.org/ 10.1371/journal.pone.0011692

10. Yaya S, Bishwajit G, Ekholuenetale M, Shah V, Kadio B, Udenlgwe $\mathrm{O}$. Knowledge of prevention, cause, symptom and practices of malaria among women in Burkina Faso. PLoS One 2017; 12 : e1080508. https://doi.org/10.1371/journal.pone.0180508

11. Deressa W, Ali A, Enquiselassie F. Knowledge, attitude and practice about malaria, the mosquito and antimalarial drugs in a rural community. Ethiop J Health Dev 2004; 17: 99-104. https:// doi.org/10.4314/ejhd.v17i2.9849

12. Munzhedzi M, McQuade ETR, Guler JL, Shifflett PE, Krivacsy S, Dillingham R, Bessong PO. CommuUIty knowledge, attitudes and practices towards malaria in Ha-LambaUI, Limpopo Province, South Africa: a cross-sectional household survey. Malar J 2021; 20: 188. https://doi.org/10.1186/s12936-021-03724-Z
13. Tesfahunegn A, Zenebe D, Addisu A. Determinants of malaria treatment delay in northwestern zone of Tigray region, Northern Ethiopia, 2018. Malar J 2019; 18: 358. https://doi.org/10.1186/ s12936-019-2992-7

14. Goshu YA, Yitayew AE. Malaria knowledge and its associated factorsamong pregnant women attending antenatal cliUIc of Adis Zemen Hospital, North-western Ethiopia, 2018. PLoS One 2019; 14; e0210221. https://doi.org/10.1371/journal.pone.0210221

15. MuUIsi DZ, Myundo AA, Mpondo BC. Knowledge, attitude and practice towards malaria among symptomatic patients attending Tumbi Referral Hospital: a cross-sectional study. PLoS One 2019; 14: e0220501. https://doi.org/10.1371/journal.pone.0220501

16. Amusan VO, Umar YA, Vantsawa PA. Knowledge, attitudes and practices on malaria prevention and control among private security guards within Kaduna. Sci J Pub Health 2017; 5: 240-245. https://doi.org/10.11648/j.sjph.20170503.22

17. Hlongwana KW, Mabaso ML, Kunene S, Govender D, Maharaj R. CommuUIty knowledge, attitudes and practices (KAP) on malaria in Swaziland: a country earnmarked for malaria elimination. Malar J 2009; 8: 29. https://doi.org/10.1186/1475-2875-8-29

18. Ndegwa DN, Kundu P, Hostetler JB, Marin-Menendez A, Sanderson T, Mwikali K, Verzier LH, Coyle, Adjalley S, Rayner JC. Using Plasmodium knowlesi as a model for screeUIng Plasmodium vivax blood-stage malaria vaccine targets reveal new candidates. PLoS Pathog 2021; 17: e1008864. https://doi.org/10.1371/journal.ppat. 1008864 
